\title{
USO DE LA TÉCNICA ROMPECABEZAS PARA PROMOVER LA HABILIDAD DE RESOLUCIÓN DE PROBLEMAS Y EL TRABAJO COLABORATIVO EN TAREAS DE EXPRESIÓN ORAL EN INGLÉS
}

\author{
Use of the Jigsaw Technique to promote problem-solving skills and \\ collaborative work in English speaking tasks
}

\author{
Cecilia Quiroga Castillo ${ }^{1}$ \\ Karin Sepúlveda Navarro ${ }^{2}$ \\ Claudio Díaz Larenas ${ }^{3}$
}

\begin{abstract}
RESUMEN
El presente artículo describe un estudio cuyo objetivo fue implementar la Técnica de aprendizaje Rompecabezas para desarrollar las habilidades cognitivas de orden superior de resolución de problemas y la habilidad colaborativa en 31 estudiantes de sexto año de educación primaria en la asignatura de inglés. Los aprendices realizaron tareas de expresión oral en inglés en grupos pequeños por dos horas semanales durante siete semanas para la unidad didáctica "En la Ciudad"; a cada uno de los participantes se les invitó a comprometerse e interactuar colaborativamente para lograr la solución de las tareas. La recolección de datos se obtuvo de la aplicación de las mediciones de diagnóstico y de término para los Grupos Rompecabezas, ambas evaluadas mediante una rúbrica analítica de resolución de problemas y una escala de estimación para el trabajo colaborativo. Se concluyó la experiencia con la realización de una encuesta de satisfacción de la Técnica Rompecabezas dirigida a los participantes. Al final, los estudiantes desarrollaron progresivamente la habilidad de resolución de problemas, que demostró, asimismo, mejoras en el trabajo colaborativo.
\end{abstract}

Palabras clave: Técnica Rompecabezas, habilidades del pensamiento de orden superior, resolución de problemas, trabajo colaborativo, expresión oral.

\begin{abstract}
This article describes a study whose objective was the implementation of the Jigsaw Learning Technique to develop the higher order problem-solving and collaborative skills on 31 sixth grade students in the English subject. Students performed English oral expression activities in small groups during two hours weekly, the unit was "Around Town" and lasted seven weeks. Every participant was engaged and interacted collaboratively to accomplish the tasks. The data collection was obtained from the application of a diagnostic task and a final evaluation task for Jigsaw groups, evaluated by a problem-solving analytical rubric and a collaborative work rating scale. The experience was concluded by the application of a Satisfaction Survey of the Jigsaw Technique. In this way, students progressively developed the problem solving skill; likewise, they demonstrated an improvement in collaborative work.
\end{abstract}

Key Words: Jigsaw Technique, higher order thinking skills, problem solving, collaborative work, speaking skills.

\footnotetext{
${ }^{1}$ Universidad de Concepción. Profesora de Inglés y magíster en Educación. Chile.

Correo electrónico: cquiroga@udec.cl

${ }^{2}$ Universidad de Concepción. Profesora de Inglés y magíster en Educación. Chile.

Correo electrónico: ksepulveda@udec.cl

${ }^{3}$ Universidad de Concepción. Profesor de inglés, magíster en Lingüística y doctor en Educación, director de Magister en Innovación de la Enseñanza, Aprendizaje y Evaluación del Inglés. Chile.

Correo electrónico: claudiodiaz@udec.cl

Recepción: 25-11-17 Aceptación: 05-03-2018
} 


\section{Introducción}

El subsector de Lengua Extranjera (inglés) considera desarrollar las habilidades de comunicación del idioma: la comprensión auditiva, la comprensión de lectura, la expresión oral y la expresión escrita, asimismo se sugiere enseñar mediante el uso del Enfoque Comunicativo. Sin embargo, generalmente, se tiende a enseñar la lengua inglesa de manera aislada, descontextualizada y sin fomentar un esfuerzo cognitivo por parte de los estudiantes, limitando sus habilidades a la mera repetición y memorización de contenidos lingüísticos.

Las motivaciones para la realización de esta investigación-acción recae en la importancia de la enseñanza del idioma inglés para que los estudiantes sean capaces de comunicar y enfrentarse a los desafíos comunicativos del mundo globalizado, por lo que se requiere desarrollar las habilidades que, según el Programa de Estudios de $6 .^{\circ}$ básico, son entendidas como "capacidades para realizar tareas y para solucionar problemas con precisión y adaptabilidad" (2012, p. 10).

Es importante un cambio en el enfoque didáctico utilizado por los docentes, que evite centrarse exclusivamente en lograr que los estudiantes dominen los conocimientos de las estructuras léxicas, gramaticales o fonológicas del idioma, o solo la comprensión de textos orales y escritos. Además, es necesario invitar a los estudiantes a participar en tareas comunicativas que permitan usar el idioma hablado de manera natural, y puedan prácticar diferentes aspectos del pensamiento para así resolver problemas. Por lo tanto, esta investigación tiene como objetivo promover el desarrollo de la habilidad de resolución de problemas y colaboración en los estudiantes, mediante la implementación de la Técnica de aprendizaje Rompecabezas en tareas de expresión oral en inglés. Este artículo se inserta en el contexto del proyecto Fondecyt 1150889, titulado Las dimensiones cognitivas, afectivas y sociales del proceso de planificación de aula y su relación con los desempeños pedagógicos en estudiantes de práctica profesional y profesores nóveles de pedagogía en inglés. 


\section{Marco teórico}

\subsection{Habilidades del pensamiento de orden superior (HOTS)}

Para Metts, el profesor tiene la responsabilidad de "cuestionarse si la clase de preguntas y tareas que usa en el aula, crean y animan un ambiente que favorezca el desarrollo de habilidades de pensamiento de orden superior" (1997, p. 246). Por lo tanto, las preguntas y tareas no deben ser para obtener respuestas cortas y memorizadas. Brookhart (2010) identifica tres categorías en las que se pueden presentar las habilidades superiores del pensamiento: transferencia, pensamiento crítico y resolución de problemas. En este sentido, transferencia corresponde al proceso y capacidad de poder recordar y usar lo aprendido, demostrando entendimiento de aquello. Para definir pensamiento crítico, la autora cita a Norris y Ennis (1989), quienes lo consideran como un "pensamiento razonable y reflexivo que se enfoca en decidir qué creer o hacer" (Brookhart, 2010, p. 4). Finalmente, la habilidad de resolución de problemas incluye procesos del pensamiento necesarios para alcanzar un objetivo esperado en la solución a un problema.

Según la taxonomía revisada de Bloom, las habilidades de orden superior se relacionan con el análisis, en donde algo se divide en sus partes para determinar cómo estas se relacionan (Woolfolk, 2006). Por otro lado, Aragón et al. (2012, p. 30) citan a Churches (2007), quien define la habilidad de evaluar como "hacer juicios en base a criterios y estándares utilizando la comprobación y la crítica". El mismo autor define crear como "juntar los elementos para formar un todo coherente y funcional; generar, planear o producir para reorganizar elementos en un nuevo patrón o estructura” (Churches, 2007, citado en Aragón et al., 2012, p. 31).

Particularmente, en este proyecto de intervención pedagógica se midió la habilidad de resolución de problemas porque permite a los estudiantes producir "negociaciones, y, por tanto, interacción de calidad" (Edwards y Willis, 2004, p. 247). Además, incita a los estudiantes a "crear significados para centrarse en el proceso de aprendizaje y del pensamiento" para ser activos (Ho, 2008, p. 108). Asimismo, Ho cita a Barrows (1985), quien afirma que los problemas involucran a los estudiantes "con un esfuerzo colaborativo, en cuatro tareas principales: comprensión del problema, aprendizaje, solución, y reflexión” (Barrows, 1985, citado en Ho, 2008, p. 4). 


\subsection{Técnica Rompecabezas (Jigsaw)}

La Técnica Rompecabezas fue propuesta originalmente por Aronson en el año 1971 en la Universidad de Texas y en la Universidad de California (Daradoumis et al., 2012). Hull (2013) da a conocer el contexto específicamente en Austin, Texas en aquella época, fundamentando que como resultado de la desegregación racial en Estados Unidos se produjo hostilidad entre los estudiantes, debido a la convivencia de diferentes razas, razón por la cual Aronson y sus colegas decidieron aplicar una técnica para reunir grupos cultural y racialmente diversos, con el fin de reducir las divisiones entre los estudiantes.

La Técnica Rompecabezas (Jigsaw) se caracteriza en educación por integrar actividades que requieren construir el conocimiento a partir de su división en diferentes partes. Este tipo de actividad surgió, además, para asegurar una comunicación genuina o auténtica en la enseñanza de idiomas (Corbett, 2003), ya que requiere el intercambio entre pares, y se sustenta en contextos reales y, por tanto, significativos, promoviendo también la fluidez en el idioma mediante un trabajo colaborativo.

Beltrán y Bueno (1995, p. 511) mencionan que la Técnica Rompecabezas "exige que el maestro divida el tema a trabajar en tantas partes con sentido, como niños vayan a existir en cada grupo de trabajo". Además, explica un posible procedimiento de la puesta en práctica de esta técnica en el aula:

1. Reunir a los alumnos en grupos para que estudien individualmente la fracción del tema que les haya correspondido.

2. Agrupar a todos los niños que tengan la subunidad 1, la 2, la 3, etc., y en estos nuevos grupos poner en común la misma información para aclarar dudas y acordar la manera de explicarlo a los del grupo de origen; el profesor aclara las preguntas solicitadas.

3. Restablecer los grupos de origen para que cada miembro explique a sus compañeros su subunidad; los demás toman notas y plantean inquietudes.

4. Otorgar instancias para que cada alumno estudie individualmente con las notas tomadas. 
5. Aplicar evaluación individual y calificación final media del equipo (Beltrán y Bueno, 1995).

Silver et al. (2007) indican que los grupos originales se denominan "Grupos Rompecabezas", los cuales pueden ser integrados heterogéneamente por 3 a 5 estudiantes, cada uno de ellos conformarán los llamados "Grupos Expertos”. Estos últimos comparten un subtema asignado y toman "la responsabilidad de convertirse en expertos" (Silver et al., 2007, p. 183) en dicha información, para así “desarrollar un plan para enseñar lo aprendido a sus Grupos Rompecabezas" (Silver et al., 2007, p. 183). Luego, los integrantes de los Grupos Expertos vuelven a sus respectivos Grupos Rompecabezas a enseñar sus subtemas y a aprender los otros subtemas de los demás miembros del Grupo Rompecabezas original, de esta manera, mediante la comunicación y el trabajo colaborativo, los estudiantes “construyen la situación completa” (Silver et al., 2007, p. 183). Finalmente, para evaluar el aprendizaje, “el profesor toma una prueba a los estudiantes” (Silver et al., 2007, p. 187), con el fin de otorgar un puntaje individual.

\section{Diseño de investigación}

El presente proyecto se basó en la metodología de la investigación-acción que, según Bausela (2004, p. 1), "supone entender la enseñanza como un proceso de investigación, un proceso de continua búsqueda”, con el fin de generar mejoras en el proceso de enseñanza y aprendizaje. Además, se desarrolló la investigación en las siguientes etapas: planificación, diagnóstico, implementación y evaluación.

\subsection{Objetivos del estudio}

1. Evaluar la habilidad de resolución de problemas y de colaboración durante el desarrollo de tareas de expresión oral modalidad Rompecabezas.

2. Determinar el grado de satisfacción de los estudiantes respecto del trabajo con la Técnica de aprendizaje Rompecabezas.

A continuación, se presentan los pasos de la intervención. 


\subsection{Paso 1: Planificación}

La etapa de planificación considera los aspectos de preparación para ejecutar la Técnica Rompecabezas, por lo tanto, se diseñaron y prepararon las acciones necesarias, tales como: la construcción de las actividades Rompecabezas para las cuatro sesiones, la Tarea de Diagnóstico para los Grupos Rompecabezas y la Tarea de Evaluación Final para los Grupos Rompecabezas con objetivos basados en el currículum nacional para fomentar las habilidades de resolución de problemas, el trabajo colaborativo y la fluidez en la habilidad de expresión oral.

\subsection{Paso 2: Diagnóstico}

La etapa de diagnóstico contempla la fundamentación del problema, la cual se llevó a cabo por la aplicación de los instrumentos Tarea de Diagnóstico para los Grupos Rompecabezas previo a la intervención.

\subsection{Paso 3: Implementación}

Esta etapa comenzó con una clase a modo de taller para dar a conocer las habilidades de pensamiento de orden superior y la Técnica Rompecabezas y, de esta manera, practicar la expresión oral con actividades niveladas y luego, progresivamente, se continuó con la unidad pedagógica de cuatro sesiones, para la cual se realizó una actividad contextualizada y cognitivamente más compleja sesión tras sesión semanalmente, bajo la Técnica Rompecabezas (Jigsaw). Estos procedimientos se evaluaron con la Rúbrica Analítica de Resolución de Problemas y la Escala de Estimación para el Trabajo Colaborativo.

\subsection{Paso 4: Evaluación}

Por último, en la etapa de evaluación se valoraron las actividades realizadas por medio del análisis de resultados y reflexión de la implementación de la investigaciónacción, junto con la identificación de los niveles de satisfacción de los estudiantes partícipes en dicho proyecto. Los instrumentos que se analizaron en esta etapa fueron las actividades realizadas durante las cuatro sesiones por los estudiantes en cada intervención: la Técnica Rompecabezas, la Rúbrica Analítica de Resolución de Problemas y la Escala de Estimación para el Trabajo Colaborativo tomada al inicio y final del proceso, y, finalmente, 
la Encuesta de Satisfacción de la Técnica Rompecabezas de los estudiantes al término de la investigación-acción.

\subsection{Sujetos del estudio}

Los sujetos del estudio fueron 31 estudiantes de sexto año básico, 13 niñas y 18 niños, todos de 11 años de edad, pertenecientes a un establecimiento educacional particular subvencionado con excelencia académica, cuya matrícula es de 375 estudiantes y cuyo índice de vulnerabilidad es de 54,3\%. El promedio general de los estudiantes al término del primer semestre fue $58,3 \%$, más alto que el año anterior; en la asignatura de inglés para el año anterior fue de 5,7 \%, mientras que actualmente creció, con un 6,2 \% para dicha materia. Los 31 participantes fueron divididos en ocho grupos, los cuales quedaron conformados por cuatro estudiantes cada uno, exceptuando uno, de solo tres estudiantes. Estos Grupos Rompecabezas fueron conformados considerando la heterogeneidad de género, de rendimiento y de comportamiento, con el fin de evitar formar solo grupos de niñas o niños, grupos de solo estudiantes avanzados o de únicamente estudiantes con bajo rendimiento, y por consiguiente, grupos de solo estudiantes con problemas disciplinarios. Esta selección fue realizada por la profesora a cargo, quien conoce las características de los estudiantes, con el objetivo de que los integrantes de cada equipo se complementaran en conocimientos del idioma, se adaptaran a distintos compañeros de trabajo, y que existiera un equilibrio que contribuyera a mantener el orden para lograr las metas de la realización de tareas grupales de la Técnica Rompecabezas.

\section{Instrumentos de recolección de la información}

\subsection{Tarea de diagnóstico para los grupos rompecabezas}

La denominada Tarea de Diagnóstico para los Grupos Rompecabezas fue la primera actividad que los estudiantes realizaron bajo el procedimiento de la Técnica Rompecabezas. Cada uno de los integrantes del grupo recibió una descripción de una persona, la cual debieron analizar de manera individual; luego los estudiantes con la misma descripción se reunieron y conformaron los Grupos Expertos para resolver dudas sobre sus partes y después volver a sus Grupos Rompecabezas a explicarlas y conocer las demás descripciones. Finalmente, los grupos recibieron la tarea por resolver, la cual consistía en 
decidir el restaurante más adecuado para cada persona y fundamentar su decisión, para esto recibieron una hoja que describía los menús de cuatro diferentes restaurantes y una hoja de respuesta para registrar su discusión y resultados de la interacción grupal.

\subsection{Tareas para los Grupos Rompecabezas en la etapa de implementación}

En la misma lógica del procedimiento de la actividad de diagnóstico, los grupos realizaron la segunda tarea de rompecabezas, la cual consistió en discutir sobre el lugar de trabajo de cada personaje según las características de las ocupaciones que analizaron. En la siguiente sesión, cada integrante de los grupos analizó la imagen de promoción de un evento en la ciudad (cine, circo, zoológico y museo) para luego leer los gustos de cuatro personas y decidir el evento ideal para ellas, entregando razones para sus respuestas. Durante la cuarta tarea de rompecabezas, cada participante analizó la descripción de un trabajador que detallaba la ubicación de su lugar de empleo en la ciudad y luego, como grupo, determinaron la ubicación de los diferentes lugares mencionados en un mapa. Finalmente, la actividad previa a la evaluación final consistió en sugerir a diferentes personajes el mejor lugar para vivir, según el mapa de una ciudad y las descripciones de preferencias de los personajes de acuerdo a sus profesiones.

\subsection{Rúbrica analítica de resolución de problemas}

Para evaluar la Tarea de Diagnóstico para los Grupos Rompecabezas se utilizó una rúbrica analítica de desempeño, la cual constaba de diez criterios y cuatro niveles de desempeño: Excelente, Bueno, Insuficiente, Nulo, que sumaban un total de treinta puntos como puntaje máximo. Esta fue construida para observar las etapas de la técnica, el comportamiento e interacción de los estudiantes y la utilización de la expresión oral del idioma inglés. Los criterios relevantes en este instrumento fueron: Análisis individual de cada subtema del rompecabezas (este evaluaba que cada integrante del grupo analizara y comprendiera la situación de la tarea); Investigación de cada subtema del rompecabezas en los Grupos Expertos (los estudiantes debían demostrar capacidad de investigación, acudiendo a diferentes fuentes de información); Discusión de la Tarea, (evaluaba que los participantes propusieran y compartieran ideas variadas para alcanzar una solución); y Acuerdo en la solución de la tarea (estimaba si los grupos producían una solución de manera consensuada). 


\subsection{Escala de estimación para el trabajo colaborativo}

Adicionalmente, se evaluó el trabajo colaborativo con una escala de estimación, la cual consistía en dos dimensiones: la Colaboración y la Participación, con los mismos cuatro niveles de desempeño de la rúbrica mencionada anteriormente. Estos dos últimos instrumentos se utilizaron también para evaluar las cuatro sesiones de intervención y la Tarea de Evaluación Final para los Grupos Rompecabezas, última evaluación. Entre los criterios que conformaron ambas dimensiones se encontraban: El grupo respetó los turnos de habla de los compañeros; Los integrantes del grupo respetaron a sus compañeros; El grupo escuchó respetuosamente las ideas y opiniones de cada integrante; y Los integrantes del grupo realizaron preguntas para resolver sus dudas.

\subsection{Tarea de Evaluación Final para los Grupos Rompecabezas}

La Tarea de Evaluación Final para los Grupos Rompecabezas corresponde a la última actividad de la intervención bajo el procedimiento la Técnica Rompecabezas. Esta fue elaborada con un formato similar a la Tarea de Diagnóstico para los Grupos Rompecabezas para propósitos comparativos de la presente investigación-acción, sin embargo, se agregó un mayor grado de dificultad en el nivel de vocabulario en inglés. En esta ocasión, los Grupos Rompecabezas debieron llegar a una solución, al elegir y fundamentar la ocupación más adecuada para cada persona según los avisos de empleos entregados y las respectivas características de las personas.

\subsection{Encuesta de satisfacción de la Técnica Rompecabezas}

Por último, se aplicó una encuesta de satisfacción de la Técnica Rompecabezas a los 31 estudiantes. Esta encuesta fue adaptada de una versión creada por Mengduo y Xiaoling (2010), la cual quedó constituida por seis dimensiones: Interdependencia positiva, Interacción cara a cara, Responsabilidad grupal e individual, Habilidades interpersonales, Procesamiento grupal y Apreciación general, cada una de estas con cuatro criterios, y con opciones de tres niveles de desempeño: De acuerdo, Medianamente de acuerdo y En desacuerdo. 


\section{Procedimientos}

\subsection{Tarea de diagnóstico para los Grupos Rompecabezas, Rúbrica Analítica de Resolución de Problemas y Escala de Estimación para el Trabajo Colaborativo}

La Rúbrica Analítica de Resolución de Problemas y la Escala de Estimación para el Trabajo Colaborativo fueron empleadas por la profesora de inglés a cargo (Evaluador 1) y una profesora General Básica con mención en inglés a cargo de la Unidad Técnico Pedagógica del establecimiento educacional (Evaluador 2) para valorar el trabajo de los ocho grupos en la primera actividad de la Técnica Rompecabezas: Tarea de Diagnóstico para los Grupos Rompecabezas. Esta tarea tuvo una duración aproximada de una hora y fue llevada a cabo en la sala de clases de sexto año básico.

Durante la intervención en sí misma, es decir, las demás cuatro sesiones de la Técnica Rompecabezas, se utilizó la misma Rúbrica Analítica de Resolución de Problemas y la Escala de Estimación para el Trabajo Colaborativo, sin embargo, solo la profesora de inglés a cargo de la investigación (Evaluador 1) evaluó.

\subsection{Encuesta de satisfacción de la Técnica Rompecabezas}

Finalmente, la Encuesta de Satisfacción de la Técnica Rompecabezas fue aplicada individualmente para concluir la experiencia y dar a conocer sus apreciaciones sobre el proceso vivido.

Los instrumentos utilizados en las etapas de diagnóstico, implementación y evaluación fueron de elaboración propia y validados por juicio de expertos, para lo cual cuatro profesores de inglés entregaron sus valoraciones y sugerencias.

\section{Procedimientos de análisis de la información}

Los procedimientos de análisis de la información utilizados fueron con estadística descriptiva para extraer la frecuencia y hacer las representaciones gráficas para los resultados obtenidos en los diferentes instrumentos aplicados durante toda la presente investigación-acción. 
En el caso de la primera parte de la Tarea de Diagnóstico para los Grupos Rompecabezas, la cual fue evaluada por el instrumento Rúbrica Analítica de Resolución de Problemas, se utilizó gráficos de columna, comparando los resultados globales de los grupos para las dos evaluadoras. Asimismo, para la evaluación del trabajo colaborativo, medido por la Escala de Estimación para el Trabajo Colaborativo se realizó un gráfico de columna para representar el desempeño por dimensión al inicio y al final de la intervención, junto con el desempeño global de todos los grupos al comienzo y al término de esta investigación-acción.

El proceso de seguimiento de los grupos evaluados durante las cuatro sesiones se representó con gráficos de línea, que mostraron el desempeño de cada grupo en cada una de las intervenciones de la Técnica Rompecabezas. Por otro lado, los resultados del instrumento Rúbrica Analítica de Resolución de Problemas para la Tarea de Evaluación Final para los Grupos Rompecabezas se representaron en gráficos de columna, que permitieron comparar los resultados globales de cada equipo para las dos profesoras evaluadoras.

En el caso de la Encuesta de Satisfacción de la Técnica Rompecabezas, las apreciaciones según grado de acuerdo de los estudiantes con los diferentes indicadores se dieron a conocer a través de gráficos de barra para cada dimensión.

Finalmente, se calculó el coeficiente Kappa para conocer el grado de concordancia entre las dos observadoras que evaluaron a los equipos al inicio y al final de la intervención de la Técnica Rompecabezas para la resolución de problemas y el trabajo colaborativo.

\section{Análisis de los datos}

\subsection{Resultados de la etapa inicial}

A continuación, se muestra una comparación de los resultados obtenidos por los ocho grupos en la Rúbrica Analítica de Resolución de Problemas, según el Evaluador 1 y Evaluador 2 (Ver Figura 1). 


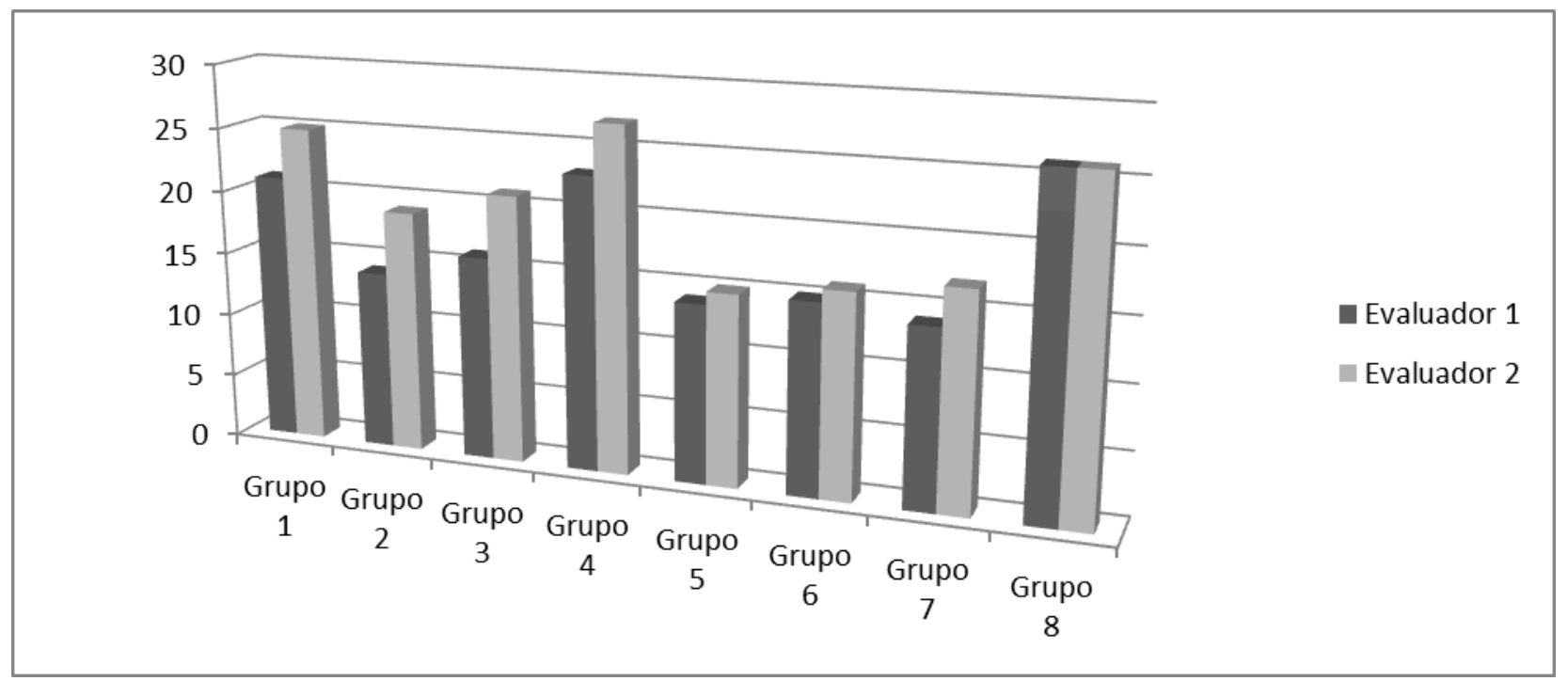

Figura 1. Desempeño global de los grupos de la Técnica Rompecabezas en la Tarea de Diagnóstico para los Grupos Rompecabezas

Tras la aplicación de la Tarea de Diagnóstico para los Grupos Rompecabezas se puede apreciar que ningún grupo logró alcanzar el puntaje máximo de treinta puntos, a pesar de esto el Grupo 4 fue el mejor evaluado con 27 puntos, según el Evaluador 2, y el Grupo 8 llegó a los 26 puntos, según ambas evaluadoras. Los grupos con resultados más bajos para el Evaluador 1 fueron el Grupo 2, el Grupo 5, el Grupo 6 y el Grupo 7, con solo 14 puntos. Estos tres últimos coinciden con la apreciación del Evaluador 2, quien también les otorga los puntajes más bajos.

Los aspectos con desempeño más bajos en todos los grupos fueron: Criterio 3: Explicación de cada subtema del rompecabezas en los Grupos Originales; Criterio 4: Interacción en el Grupo Rompecabezas; Criterio 6: Discusión de la tarea; Criterio 8: Acuerdo en la tarea; y, sobre todo, en el Criterio 10: Expresión oral, para el cual todos los grupos obtuvieron solo un punto, ya que rara vez comunicaban sus ideas en inglés. Finalmente, se aprecian semejanzas en las evaluaciones realizadas para cada grupo, difiriendo en 5 puntos para los Grupos 2 y 3, y en 4 puntos para los Grupos 1 y 4.

Los resultados de la Escala de Estimación para el Trabajo Colaborativo serán presentados en el apartado: Resultados de la Etapa Final, donde se comparan los resultados globales del inicio y del final de la intervención. 
A continuación, se presentan los resultados del coeficiente Kappa calculados con el programa estadístico IBM SPSS Statistics 19 para la Rúbrica Analítica de Resolución de Problemas y la Escala de Estimación para el Trabajo Colaborativo que evaluaron la Tarea de Diagnóstico para los Grupos Rompecabezas. Cerda y Villarroel mencionan que el llamado coeficiente Kappa es un test que "refleja la fuerza de la concordancia entre dos observadores”, es usado con más frecuencia para datos categóricos, y su fórmula incorpora "una corrección que excluye la concordancia atribuible al azar, permitiendo una estimación más precisa de la concordancia genuina" (Cerda y Villarroel, 2008, p. 56). Estos autores informan que este coeficiente puede tomar valores entre $-1 \mathrm{y}+1$, si su valor se acerca $\mathrm{a}+1$, el grado de concordancia inter-observador será mayor; al contrario, si su valor se acerca a 1, el grado de discordancia inter-observador será mayor.

En relación con los resultados obtenidos por ambos evaluadores en la Tarea de Diagnóstico para los Grupos Rompecabezas en la resolución de problemas en la Técnica Rompecabezas, se aprecia que el nivel de concordancia inter-observadores calculado por el Coeficiente Kappa es de fuerza leve para los Grupos 2 y 8, ya que sus valores se encuentran entre 0,01 y 0,20; es considerada aceptable para los Grupos 1, 3, 4 y 6, ya que sus valores están entre 0,21 y 0,40; y presenta mayor fuerza para el Grupo 7, la cual corresponde al nivel moderada, debido a que su valor está entre 0,41 y 0,60 . Por otro lado, la concordancia para el Grupo 5 se considera pobre, ya que su valor es 0,00 . Con respecto a la concordancia entre ambos observadores para la evaluación del trabajo colaborativo por equipo, se percibe en orden ascendente de fuerza, una concordancia aceptable para los Grupos 4, 5 y 8, en cuanto a que sus valores se encuentran entre 0,21 y 0,40 ; moderada para los Grupos 1, 2, 6 y 7, ya que asumen valores entre 0,41 y 0,60; mientras que para el Grupo 3 se determina considerable por su valor situado entre 0,61 y 0,80 .

\subsection{Resultados en la etapa de seguimiento}

Los grupos fueron evaluados por la profesora de inglés a cargo para realizar el seguimiento de estos durante las cuatro sesiones de intervención, utilizando la Rúbrica Analítica de Resolución de Problemas para evaluar el procedimiento de la técnica; y la Escala de Estimación para el Trabajo Colaborativo de los equipos. A continuación, se 
presentan los resultados globales obtenidos por los Grupos Rompecabezas en las cuatro sesiones (Ver Figura 2).

Tras las cuatro sesiones de implementación, se puede apreciar una mejora en los resultados de los grupos, especialmente los Grupos 5 y 7, los cuales solo comenzaron con 17 puntos en la primera sesión, terminando ambos con 28 puntos de un total de 30 en la última sesión. Además, se aprecia que los grupos que obtuvieron los mejores puntajes desde el inicio fueron el Grupo 8 con 27 puntos, manteniéndose y alcanzando el puntaje máximo en la cuarta sesión; y el Grupo 4, el cual comenzó con 24 puntos, logrando alcanzar también el puntaje máximo en la última sesión. Por otro lado, a pesar de demostrar un avance durante la implementación, los Grupos 2 y 3 fueron evaluados con 27 puntos, los Grupos 5, 6 y 7 alcanzaron 28 puntos y el Grupo 1 logró 29 puntos. Finalmente, se aprecia un alza en el desempeño de los grupos desde la sesión 3 a la sesión 4.

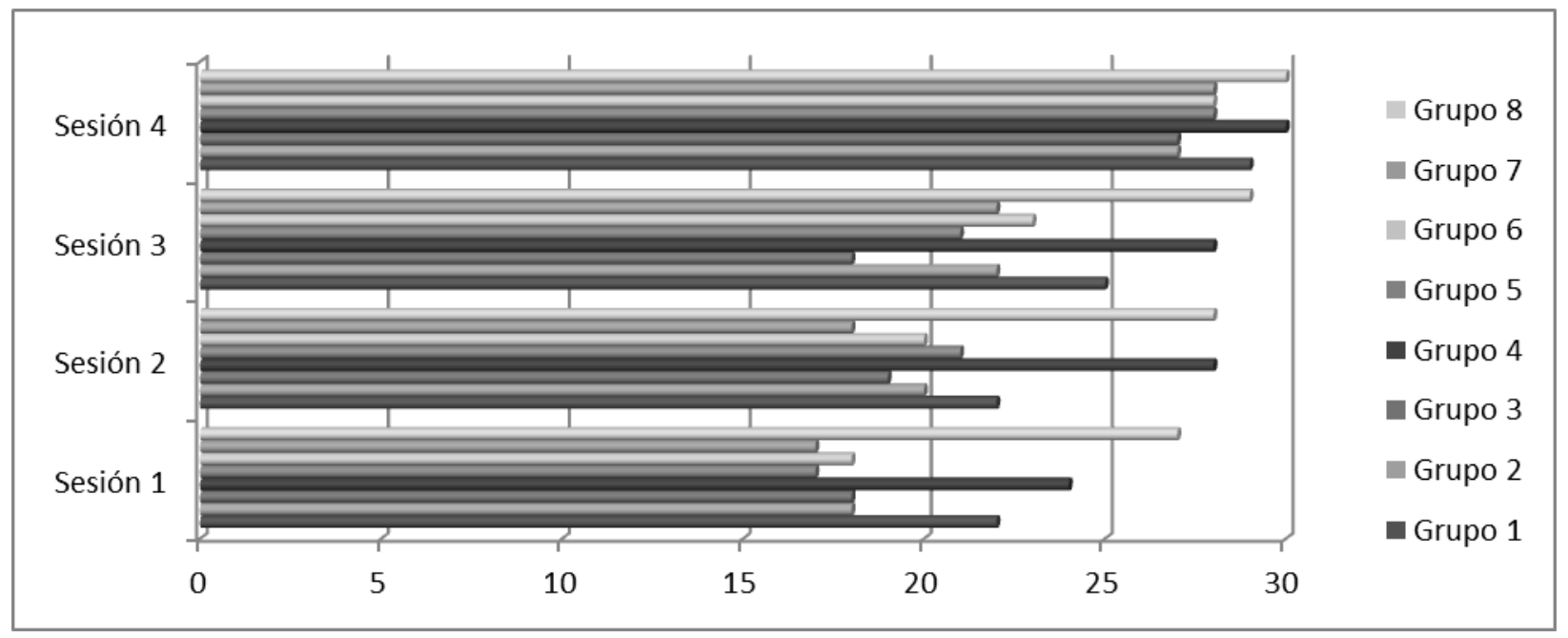

Figura 2. Desempeño global de los grupos de la Técnica Rompecabezas en las cuatro sesiones de implementación

En relación al trabajo colaborativo demostrado en las cuatro sesiones, se destacan los Grupos 4 y 8, con 50 y 52 puntos respectivamente en la sesión 1, que concluyen con los 57 puntos máximos en la sesión 4. Los grupos que demostraron más progreso fueron los Grupos 2 y 7, quienes avanzaron 20 puntos desde la primera sesión hasta la última. En 
síntesis, al comparar los puntajes totales obtenidos sesión tras sesión, todos los grupos lograron mejorar en los aspectos evaluados para el trabajo colaborativo durante las sesiones de intervención.

\subsection{Resultados de la etapa final}

Los resultados de la etapa final están relacionados con los instrumentos de Rúbrica Analítica para la Resolución de Problemas, Escala de Estimación para el Trabajo Colaborativo y Encuesta de Satisfacción de la Técnica Rompecabezas.

Tras la aplicación de la Tarea de Evaluación Final para los Grupos Rompecabezas se aprecia que la mayoría de los grupos obtuvo puntajes altos en la Rúbrica Analítica de Resolución de Problemas y únicamente dos equipos alcanzaron el puntaje máximo; sin embargo, solo uno por parte de ambas evaluadoras, el Grupo 8. El grupo que obtuvo el desempeño más bajo fue el Grupo 2 por el Evaluador 2, con solo 23 puntos; a pesar de esto, el Evaluador 1 le otorgó 27 puntos, de igual manera se identifica como el puntaje más bajo entregado por el primer evaluador. En resumen, se aprecia que el Evaluador 1 otorgó los puntajes más altos a la mayoría de los equipos, exceptuando el Grupo 7.

\subsubsection{Escala de estimación para el trabajo colaborativo}

Por medio de la comparación de los resultados de la Escala de Estimación para el Trabajo Colaborativo, con la cual se evaluó el trabajo en equipo de los Grupos Rompecabezas durante la Tarea de Diagnóstico para los Grupos Rompecabezas y la Tarea de Evaluación Final para los Grupos Rompecabezas realizada por ambas evaluadoras, se aprecia que el desempeño para la Dimensión 1: Colaboración, subió 11 puntos de 18,9 puntos a 30, mientras que la Dimensión 2: Participación, solo mejoró en casi 5 puntos, no alcanzando el puntaje máximo de 27 puntos. Finalmente, al considerar el desempeño global de los ocho equipos en el trabajo colaborativo, se evidencia un alza de 16 puntos al término de la intervención, donde solo faltaron 2,2 puntos para lograr el máximo de 57 puntos de esta evaluación.

A continuación, se presentan los resultados del coeficiente Kappa calculados con el programa estadístico IBM SPSS Statistics 19 para la Rúbrica Analítica de Resolución de Problemas y la Escala de Estimación para el Trabajo Colaborativo que evaluaron la Tarea de Evaluación Final para los Grupos Rompecabezas. 
El nivel de concordancia inter-observadores calculado por el Coeficiente Kappa en la evaluación final de la resolución de problemas en la Técnica Rompecabezas entrega como resultados: una discordancia para los Grupos 1 y 2, ya que sus valores están cercanos a -1; una concordancia leve para el Grupo 3, porque su valor está entre 0,01 y 0,20; una concordancia considerable para los Grupos 5, 6 y 7, en los cuales se presentan, coincidentemente, valores entre 0,61 y 0,80; finalmente, la concordancia para los Grupos 4 y 8 no fue calculada por el programa estadístico IBM SPSS Statistics 19, debido a que representa una constante, es decir, ambos evaluadores arrojaron los mismos valores y, por tanto, se puede considerar como una concordancia de fuerza perfecta.

El nivel de concordancia inter-observadores calculado por el Coeficiente Kappa en la evaluación final del trabajo colaborativo en los Grupos Rompecabezas entrega como resultados: una concordancia considerada pobre para el Grupo 2, ya que su valor es 0,00; una concordancia aceptable para los Grupos 1 y 3, debido a que sus valores se encuentran entre 0,21 y 0,40; una concordancia denominada considerable para los Grupos 5 y 6, con valores entre 0,61 y 0,80; una concordancia casi perfecta para el Grupo 7, ya que su valor está entre 0,81 y 1,00; y, por último, la concordancia para los Grupos 4 y 8 no fue calculada por el programa estadístico IBM SPSS Statistics 19, debido a que representa una constante, es decir, ambos evaluadores lanzaron los mismos valores y, por tanto, se puede considerar como una concordancia de fuerza perfecta.

En síntesis, el nivel de concordancia inter-observadores es diferente para cada equipo, que disminuye o aumenta la fuerza al comparar los resultados de la etapa de diagnóstico y de la etapa final de la Técnica Rompecabezas, sin embargo, mayoritariamente, este nivel tiende a aumentar al término de la intervención.

\subsubsection{Encuesta de satisfacción de la Técnica Rompecabezas}

Los resultados de la Encuesta de Satisfacción de la Técnica Rompecabezas para la primera dimensión demuestran que el $97 \%$ considera importante resolver las tareas en equipo, el $87 \%$ indica que se sienten más responsables cuando trabajan bajo la técnica y consideran que aprender inglés depende de ellos mismos. El 77 \% cree que cada integrante del equipo es indispensable para el éxito grupal, y solo el $23 \%$ está medianamente de 
acuerdo con esta situación. No se aprecian estudiantes que estén en desacuerdo con los indicadores que esta dimensión propone.

En la segunda dimensión, se destaca que el $87 \%$ cree que las clases en la Técnica Rompecabezas son más participativas que las clases regulares, mientras que solo un $3 \%$ no lo considera así. Además, un 74 \% señala aprender mejor cuando trabaja en grupos, un 68 $\%$ disfruta de la interacción con sus compañeros y un $61 \%$ indica que esta técnica les da más oportunidades de hablar en inglés con sus pares (Ver Figura 3).

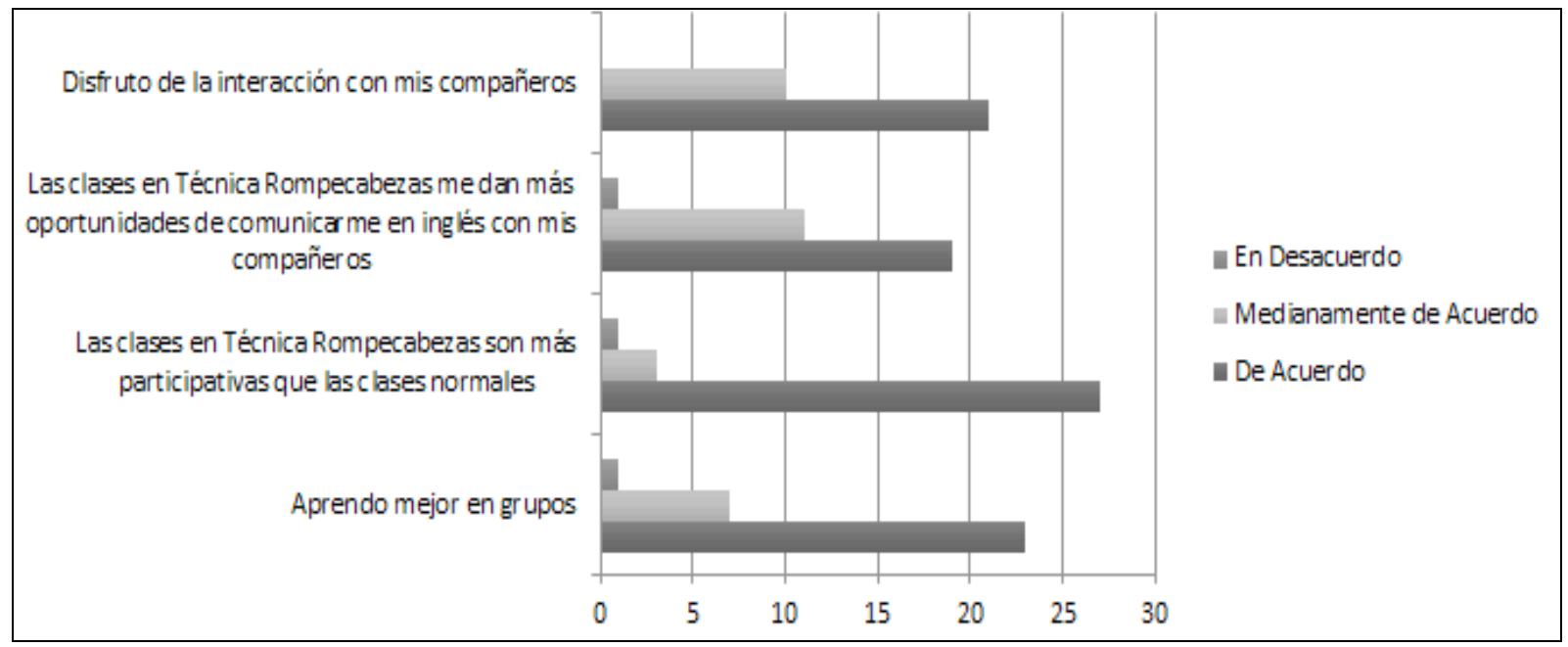

Figura 3. Encuesta de Satisfacción de la Técnica Rompecabezas: Dimensión II: Interacción cara a cara

De las respuestas para la tercera dimensión, el $94 \%$ de los estudiantes indica que es necesario verificar que todos los integrantes del equipo entiendan la tarea y que ser parte de un Grupo Rompecabezas les ayuda a mejorar su aprendizaje del inglés, sin embargo, un estudiante $(3 \%)$ está en desacuerdo con esta última declaración. El $77 \%$ señala que le gusta compartir lo que aprenden con su grupo y el $74 \%$ afirma que es importante que los grupos sean variados (Ver Figura 4). 


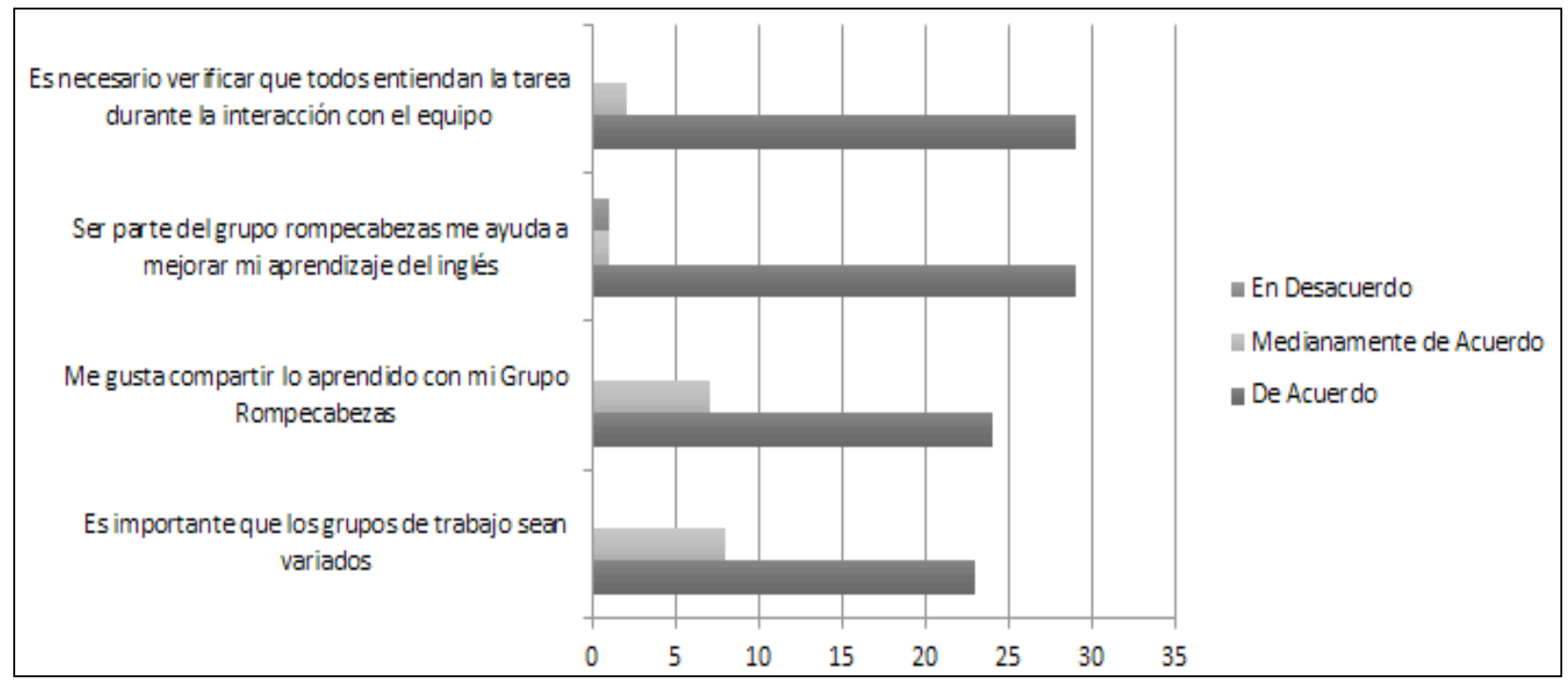

Figura 4. Encuesta de Satisfacción de la Técnica Rompecabezas: Dimensión III. Responsabilidad grupal e individual

Con respecto a la dimensión relacionada con habilidades interpersonales, los estudiantes en un $84 \%$ sostienen que les interesa lo que sus compañeros quieren enseñarles durante las actividades rompecabezas y que han aprendido a trabajar con otros pares durante el proceso de intervención. El $81 \%$ asegura que aprenden a escuchar a sus compañeros cuando trabajan en equipos y el $65 \%$, que aprenden a motivar a sus pares para trabajar; sin embargo, el 35 \% solo está medianamente de acuerdo con esto (Ver Figura 5).

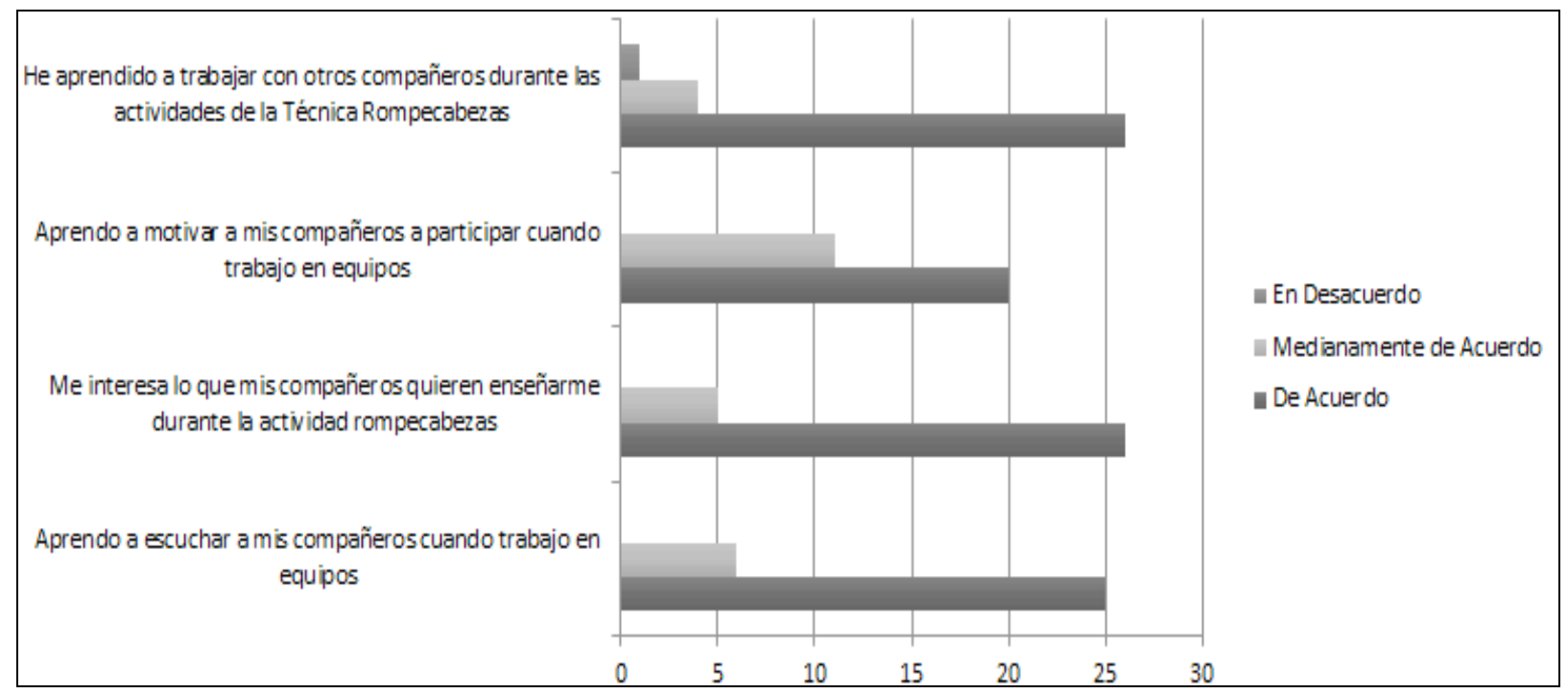

Figura 5. Encuesta de Satisfacción de la Técnica Rompecabezas: Dimensión IV. Habilidades interpersonales 
Según las apreciaciones de los estudiantes, el $77 \%$ afirma que las clases en la Técnica Rompecabezas han mejorado su comprensión sobre temas de la unidad didáctica estudiada y que también han ayudado para poder proponer ideas con el fin de resolver problemas. Además, el $65 \%$ sostiene que esta técnica hace que quieran aprender más sobre los temas tratados, mientras que el $35 \%$ no está totalmente de acuerdo con esto. Por último, el $90 \%$ señala que es importante compartir ideas para lograr la meta del equipo. No se aprecian estudiantes que estén en desacuerdo con los indicadores presentes en la dimensión de la encuesta de satisfacción (Ver Figura 6).

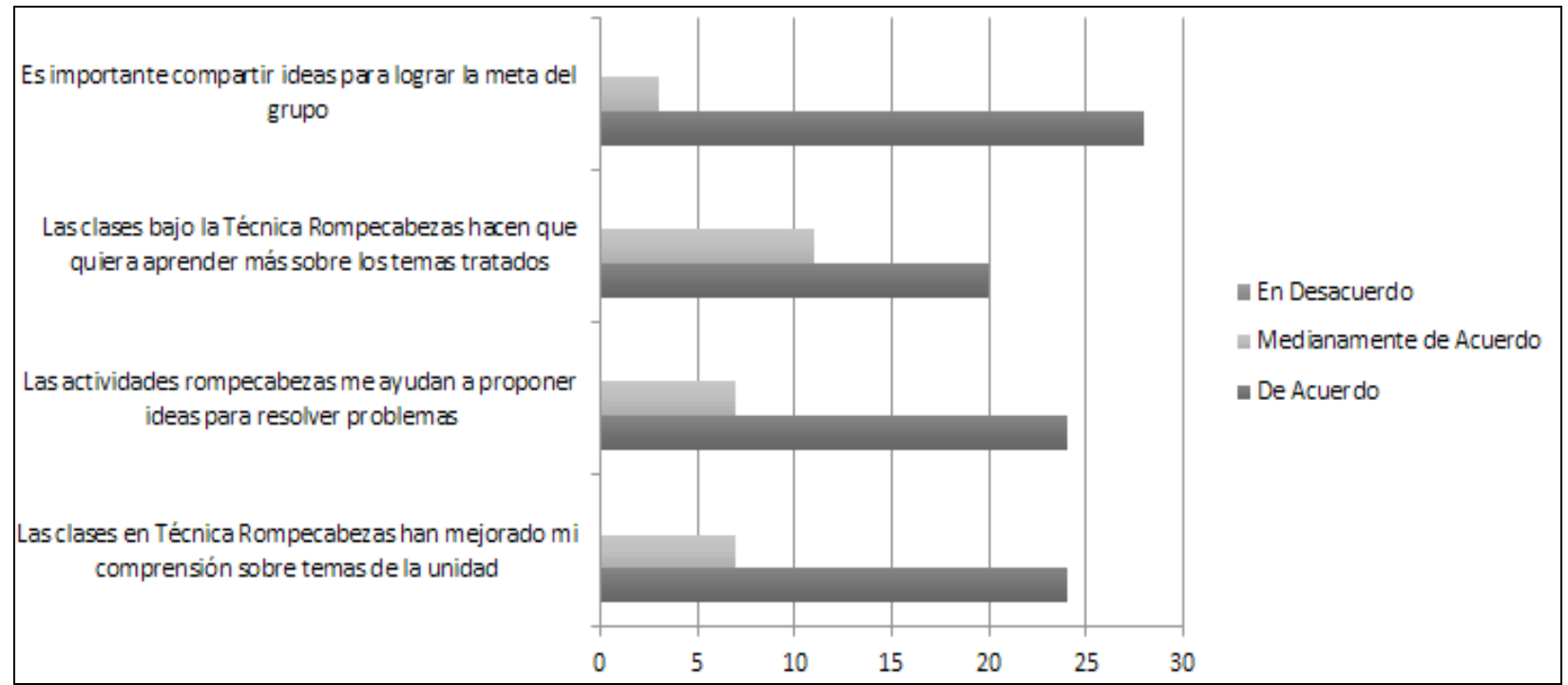

Figura 6. Encuesta de Satisfacción de la Técnica Rompecabezas: Dimensión V. Procesamiento grupal

Para la última dimensión de la Encuesta de Satisfacción de la Técnica Rompecabezas, los estudiantes dieron a conocer su grado de acuerdo para aspectos de apreciación general para esta técnica, sosteniendo en un $81 \%$ estar satisfechos con los temas y materiales utilizados, para el cual el $19 \%$ demuestra estar medianamente de acuerdo con esto. El 97 \% dice que la técnica los motiva a aprender inglés, el $58 \%$ afirma que los mantiene atento y pensando durante toda la clase, mientras que el $39 \%$ dice estar medianamente de acuerdo con esto último. Finalmente, el $77 \%$ cree que la Técnica Rompecabezas debe ser utilizada nuevamente en la asignatura de inglés y en las demás materias, y el 19 \% está medianamente de acuerdo con esto (Ver Figura 7). 


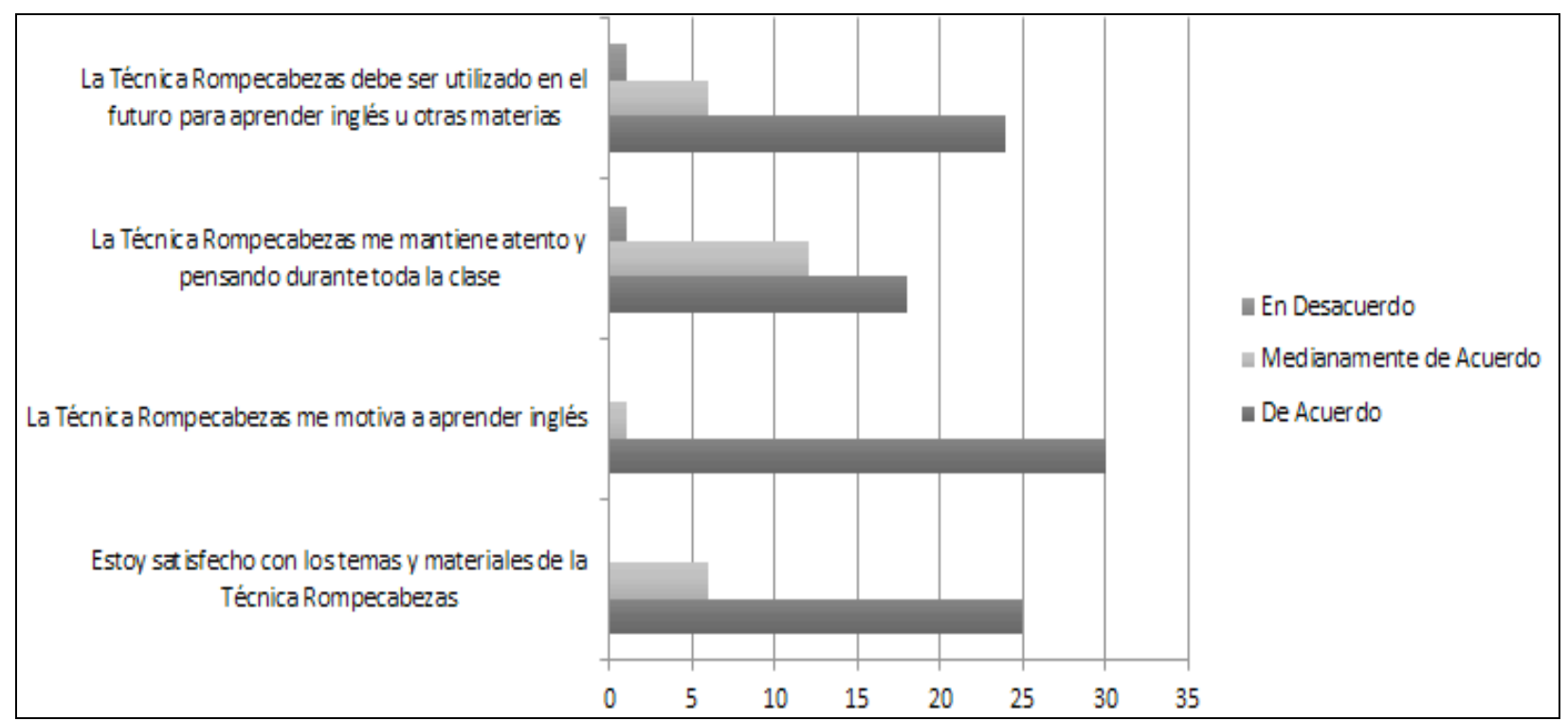

Figura 7. Encuesta de Satisfacción de la Técnica Rompecabezas: Dimensión VI. Apreciación general

\section{Discusión}

Los resultados obtenidos en la presente investigación-acción demuestran concordancia con la información entregada en los referentes teóricos, pues tratan directamente las causas del problema al: (1) alejarse de un enfoque centrado en el profesor, (2) al enfatizar la resolución de problemas comunicativos, la cual representa una habilidad de pensamiento superior, y (3) al motivar a los estudiantes a solucionar las situaciones propuestas con temas variados para la unidad de aprendizaje. Asimismo, se reflejó el bajo dominio de la expresión oral del idioma extranjero inglés, sin embargo, la Técnica Rompecabezas proporcionó la oportunidad indicada para garantizar una interacción más genuina, a lo que los estudiantes beneficiarios respondieron de manera favorable, intentando hablar en inglés con sus compañeros con mayor frecuencia, según la apreciación declarada por los participantes en la Encuesta de Satisfacción de la Técnica Rompecabezas relacionada con criterios tales como: "Las clases en Técnica Rompecabezas son más participativas que las clases normales", donde manifiestan explícitamente: "Me dan más oportunidades de comunicarme en inglés con mis compañeros". Tal como lo indica Edwards y Willis (2004), se proporciona una interacción de calidad, por lo que los educandos no adquieren el idioma a través de la repetición de palabras o diálogos 
memorizados. Por lo tanto, la propuesta de esta intervención podría ser de utilidad para mejorar el nivel de dominio en el inglés, ya que las actividades rompecabezas representan cierta complejidad que incentiva el pensamiento y la generación de ideas propias, y a la vez influyen en la mejora del rendimiento académico. Esto se corrobora con las respuestas cualitativas entregadas por los estudiantes, a saber: "Ser parte del grupo rompecabezas me ayuda a mejorar mi aprendizaje del inglés"; "Las clases en Técnica Rompecabezas han mejorado mi comprensión sobre temas de la unidad"; "Las actividades rompecabezas me ayudan a proponer ideas para resolver problemas"; "Las clases bajo la Técnica Rompecabezas hacen que quiera aprender más sobre los temas tratados"; y "Me motiva a aprender inglés".

Terminada esta intervención, se pudieron apreciar también los siguientes aspectos, que indica Barrows (1985), citado por Ho (2008): el esfuerzo colaborativo mediante la comprensión de un problema, el aprendizaje obtenido, la solución acordada y la reflexión grupal de los estudiantes en cada sesión de manera independiente. Además, se observó, en la Encuesta de Satisfacción de la Técnica, progreso en aspectos como el respeto de turnos al hablar y escuchar a sus pares, dándose cuenta de que cada uno tenía información relevante y que podían aportar; asimismo, se pudo notar el desarrollo de la autonomía, ya que cada grupo buscaba la forma de tomar nota de los compañeros y así avanzar en las actividades a su propio ritmo. Dicho progreso se reflejó en la mejora de los grupos en criterios de la encuesta, tales como: "Cada integrante es indispensable para el éxito del grupo"; "Creo que aprender inglés depende de mí mismo"; "Aprendo a escuchar a mis compañeros cuando trabajo en equipos"; y "Me siento más responsable en las actividades de la Técnica Rompecabezas". De este mismo modo, los Grupos Expertos, aquellos que compartían el mismo subtema, se ayudaban mutuamente para que todos lograran comprender su información, al complementar lo que cada uno pudo entender en la primera etapa de análisis individual, demostrando menor dependencia de la profesora a medida que avanzaba la intervención. Por lo tanto, la Técnica Rompecabezas benefició la capacidad de investigación para solucionar problemas, aprovechando fuentes como el diccionario, compañeros y docente, tal como se evidenció en el criterio Investigación de cada subtema del rompecabezas en los Grupos Expertos de la Rúbrica Analítica de Resolución de Problemas, el cual logra ser evaluado como: "Los integrantes siempre demuestran 
capacidad de investigación, preguntando dudas a compañeros de los Grupos Expertos y acudiendo a fuentes de información como el diccionario para resolverlas".

A pesar de todos los beneficios que la implementación de la Técnica Rompecabezas conllevó, es necesario tomar precauciones para evitar que las posibles situaciones complejas presentadas al principio de la intervención tengan consecuencias negativas, perjudicando la obtención de resultados positivos. Por ejemplo, el docente debe asegurar que los estudiantes entiendan de manera correcta la idea de la técnica, ya que pueden confundir la noción de que cada uno debe responder sólo la parte que le corresponde, sin interactuar ni proponer ideas para trabajar realmente de manera colaborativa. Además, se requiere que los espacios del aula destinados para conformar los grupos sean los apropiados, considerando el nivel de conocimientos y el comportamiento que los estudiantes tienen para evitar que los grupos queden conformados de manera inequitativa.

En síntesis, la implementación de la Técnica Rompecabezas puede traer muchos beneficios para los estudiantes y el docente, pero se deben considerar aspectos importantes para asegurar el éxito, tales como la conformación de los grupos, la ausencia de los estudiantes, problemas disciplinarios, conflictos entre pares y los espacios necesarios, de igual manera, se debe incluir tareas diversas para evitar que la técnica de trabajo se vuelva rutinario y predecible, perjudicando la adquisición de toda la variedad de habilidades útiles para los estudiantes proporcionadas por esta técnica.

\section{Conclusiones}

A continuación, se presentarán los principales resultados y descubrimientos de la presente investigación-acción según cada uno de los objetivos planteados.

\subsection{Objetivo de investigación 1: Evaluar la habilidad de resolución de problemas y de colaboración durante el desarrollo de tareas de expresión oral modalidad Rompecabezas}

Los resultados obtenidos en la Tarea de Diagnóstico para los Grupos Rompecabezas de implementación de la Técnica Rompecabezas se destacaron principalmente por el bajo desempeño en los criterios de Explicación de cada subtema del rompecabezas, ya que no 
todos analizaron su subtema y no todos los integrantes de los grupos lograron comprenderlos; el criterio de Interacción en el Grupo Rompecabezas, ya que los estudiantes no interactuaron solo centrados en la resolución de la tarea; el criterio Discusión de la tarea también estuvo débil, puesto que los integrantes no mostraban una tendencia a proponer y compartir ideas para solucionar la tarea; asimismo, el criterio Acuerdo en la solución de la tarea, pues, generalmente, no todos los miembros de los equipos decidieron y produjeron la solución de la tarea en consenso; y, finalmente, el criterio de Expresión Oral obtuvo en todos los grupos resultados bajos, debido a que los estudiantes no hablaron en inglés o rara vez lo hicieron. Por consiguiente, en esta etapa únicamente dos grupos obtuvieron puntaje sobre los 25 puntos de un máximo de 30. Sin embargo, al finalizar, dos grupos alcanzaron el puntaje máximo, un grupo fue considerado el más bajo con 23 puntos, mientras que los demás fluctuaron entre los 25 y 29 puntos.

Los resultados del Coeficiente Kappa para la evaluación inicial de la resolución de problemas en la Técnica Rompecabezas tuvo su fuerza más débil la considerada como "pobre" y la más alta fue la llamada "moderada". En comparación, los cálculos del coeficiente para la evaluación final resultó en una discordancia para dos grupos, pero a su vez una concordancia "perfecta" para otros dos equipos.

Con respecto a la evaluación del trabajo colaborativo, se obtuvieron resultados bajos generalizados en aspectos relacionados con la integración de compañeros y la motivación para hablar, compartir la misma responsabilidad en la tarea, escuchar respetuosamente otras ideas y, principalmente, discutir solo temas relacionados con la tarea. A pesar de esto, en la Escala de Estimación para el Trabajo Colaborativo desde la etapa de diagnóstico a la etapa de evaluación final se evidenció un alza desde los 38, 8 puntos a los 54, 8 de un total de 57 .

En relación con los niveles de concordancia del Coeficiente Kappa obtenidos para la evaluación del trabajo colaborativo, al inicio fueron "aceptable" el nivel más bajo y "considerable" el más alto. Sin embargo, los niveles de concordancia inter-observadores para la evaluación final fueron de fuerza "pobre" el más bajo y de fuerza "perfecta" el más alto.

Al finalizar la intervención se pudo apreciar el avance que experimentaron los equipos en comparación con la Tarea de Diagnóstico para los Grupos Rompecabezas, a 
pesar de esto no todos lograron el desempeño máximo por motivos, tales como: todos los integrantes proponen y comparten ideas para solucionar la tarea, uso del idioma inglés siempre o lo más posible para interactuar con compañeros, discutir solo temas relacionados con la tarea y el mantener la disciplina durante toda la actividad.

\subsection{Objetivo de investigación 2: Evaluar el grado de satisfacción de los estudiantes respecto del trabajo con la Técnica de aprendizaje Rompecabezas}

Finalmente, la Encuesta de Satisfacción de la Técnica Rompecabezas concluyó positivamente que para los estudiantes beneficiarios representó un cambio en la percepción de trabajar colaborativamente, ya que el 97 \% consideró importante resolver las actividades en equipo, reconociendo un $77 \%$ la indispensabilidad de cada integrante, y sintiendo más responsabilidad en su aprendizaje (87\%). Además, los estudiantes apreciaron las instancias de la técnica ya que el $87 \%$ cree que son más participativas, el $68 \%$ disfruta la interacción y $61 \%$ de la oportunidad de hablar en inglés, aprendiendo a trabajar con otros $(81 \%)$ y a resolver problemas $(77 \%)$.

En conclusión, esta investigación-acción implicó dar a conocer a los estudiantes una nueva forma de aprender, demostrando estar más activos en clases, proporcionando más instancias de participación y uso del pensamiento. Asimismo, aprendieron a apreciar la Técnica Rompecabezas y su utilidad para el aprendizaje del idioma inglés, desarrollando sus habilidades para proponer ideas y resolver problemas, trabajar colaborativamente y ser más responsables e independientes.

Como sugerencias para los posibles próximos ciclos se puede considerar la idea de que los Grupos Rompecabezas expongan sus soluciones a toda la clase para que los propios estudiantes puedan comparar sus resultados. Además, se puede incluir la evaluación individual al término de cada actividad rompecabezas o de la unidad pedagógica, permitiendo que los Grupos Expertos propongan las preguntas de esta evaluación. Adicionalmente, se podría trabajar las habilidades de metacognición, proporcionando instancias para la reflexión luego de cada sesión y, finalmente, en otro ciclo correspondería ampliar la cobertura de la técnica hacia otros cursos y asignaturas.

Los problemas que aún quedan por abordar, aunque estos se presentan de manera leve, serían fomentar aún más el uso hablado del inglés, tratando de limitar al mínimo el 
uso del idioma nativo a través de la entrega de frases complementarias que los estudiantes podrían utilizar para expresar ideas más completas; igualmente, los problemas disciplinarios, a pesar de ser casos específicos, podrían solucionarse rotando a los grupos y variando a los integrantes de estos, ya que quizás con otros compañeros se sientan más cómodos y motivados para trabajar, complementando las fortalezas y las debilidades del otro en inglés.

\section{Bibliografía}

Aragón, Francisco, Asensio, Fermín, Espín, María, Fernández, Adela, García, José, González, María, ... Sobrino, Diego. (2012). Aulas del siglo XXI: retos educativos. España: Maravillas Díaz Gómez.

Bausela, Esperanza. (2004). La docencia a través de la investigación-acción. Revista Iberoamericana de Educación, 20, 7-36. Recuperado de http://rieoei.org/deloslectores/682Bausela.PDF

Beltrán, Jesús y Bueno, José. (1995). Psicología de la educación. Barcelona: Editorial Boixareu Marcombo.

Brookhart, Susan. (2010). How to assess higher-order thinking skills in your classroom. Virginia: ASCD.

Cerda, Jaime y Villarroel del Pino, Luis. (2008). Evaluación de la concordancia interobservador en investigación pediátrica: Coeficiente de Kappa. Revista Chilena de Pediatría, 79(1), 54-58. Recuperado de www.scielo.cl/pdf/rcp/v79n1/art08.pdf

Corbett, John. (2003). An intercultural approach to English language teaching. United Kingdom: Multilingual Matters LTD.

Daradoumis, Thanasis, Caballé, Santi, Juan, Angel y Xhafa, Fatos. (2012). Technologyenhanced systems and tools for collaborative learning scaffolding. Catalonia: Springer.

Edwards, Corony y Willis, Jane. (2004). Teachers exploring tasks in English language teaching. United Kingdom: Palgrave McMillan. 
Ho, Wenyi. (2008). An exploration of peer collaboration and group problem solving process in a college problem-based learning classroom (Tesis doctoral). Universidad Estatal de Pensilvania.

Hull, Francis. (2013). Using jigsaw technique as an effective way of promoting cooperative learning among primary six pupils in Fijai. International Journal of Education and Practice, 1(6), 64-74. Recuperado de http://www.pakinsight.com/journals/IJEP.htm

Mengduo, Qiao y Xiaoling, Jin. (2010). Jigsaw strategy as a cooperative learning technique: focusing on the language learners. Chinese Journal of Applied Linguistics (Bimonthly), 33(4), 113-125. Recuperado de http://www.celea.org.cn/teic/92/10120608.pdf

Metts, Ralph. (1997). Ignacio lo sabia: la pedagogía jesuita y las corrientes educativas actuales. Washington, D.C.: Jesuit Secondary Education Association.

Ministerio de Educación de Chile. (2012). Idioma extranjero: Inglés Programa de Estudio para Sexto Año Básico. Recuperado de http://www.curriculumenlineamineduc.cl/605/articles-20549_programa.pdf

Silver, Harvey, Strong, Richard y Perini, Matthew. (2007). The strategic teacher. New Jersey: Pearson Education, Inc.

Woolfolk, Anita. (2006). Psicología educativa. Ohio: Pearson Educación.

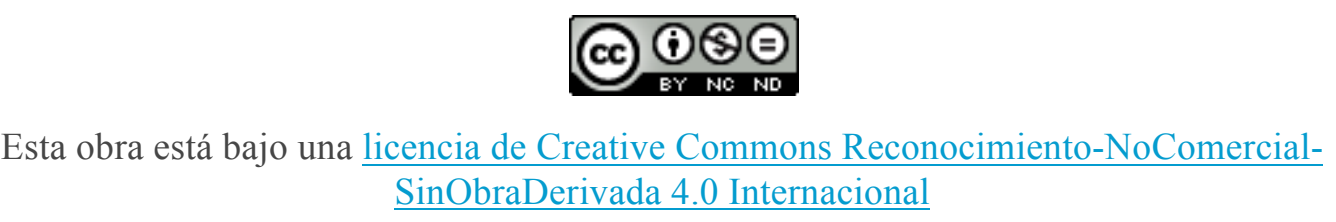

